\title{
STATIC AND DYNAMIC SPIN ORGANIZATION IN MAGNETIC SEMICONDUCTOR NANOSTRUCTURES
}

\author{
N. Samarth, H. Luo, J.K. Furdyna, T.M. Giebultowicz
}

Department of Physics, University of Notre Dame, Notre Dame IN 46556, USA

AND D.D. AwsChaLom

Department of Physics, University of California, Santa Barbara CA 93106, USA

We describe experiments which study static and dynamic aspects of $\mathrm{Mn}$ spin organization in magnetic semiconductor superlattices and quantum wells. Neutron diffraction studies of $\mathrm{ZnSe} / \mathrm{MnSe}$ and $\mathrm{ZnTe} / \mathrm{MnSe}$ superlattices show how static Mn spin organization in these antiferromagnetic layers may be modified by strain and lowered dimensionality. We also use a novel form of femtosecond magnetic spectroscopy to examine magnetic polaron dynamics in diluted magnetic semiconductor quantum wells, and show how the organization of $\mathrm{Mn}$ spins by a spin-polarized carrier population may be directly viewed in the time domain.

PACS numbers: 75.50.Pp, 75.25. $+\mathrm{z}$

\section{Introduction}

The past decade has witnessed rapid advances in the development of sophisticated vacuum deposition techniques such as molecular bcam cpitaxy (MBE) and atomic layer epitaxy (ALE). This has provided rescarchers with a variety of epitaxially layered semiconductor [1] and magnetic materials [2] which display new phenomena as a consequence of reduced dimensionality. Among the numerous nanostructures fabricated so far, those incorporating magnetic semiconductors (MS) constitute a unique nexus between the worlds of quantum semiconductor physics and thin film magnetism. The most extensively studied MS nanostructures are derived from the II-Mn-VI family [3]. From the viewpoint of pure magnetism, these materials provide archetypal examples of frustrated multilayer IIeisenberg antiferromagnets [4] and spin glasses [5] dominated by short-ranged $d-d$ exchange. The magnetic properties of MS nanostructures may be systematically manipulated through control of parameters such as dimensionality $[5,6]$, strain $[6,7]$, and dilution [8]. Further, the semiconductor nature of these systems may be exploited to tailor carrier wave functions through the use of different quantum well profiles, 
hence giving us a new way to control the $s p-d$ exchange interaction between charge carriers and Mn ions. This allows us to study novel spin-dependent phenomena such as quantum confined magnetic polarons [9] and spin-superlattices [10]. In this paper, we show how the degrees of freedom available in MS nanostructures enable us to gain insight into both static and dynamic aspects of spin organization in low dimensional magnetic systems.

\section{Static spin organization: strain-engineered antiferromagnetism}

The face centered cubic (fcc) Heisenberg antiferromagnet with short-ranged exchange is a problem which first attracted theoretical attention several decades ago [11], and still continues to be of contemporary interest because the frustration inherent to the fcc lattice leads to a rich variety of magnetic phases [12]. The fabrication by MBE of structures containing the zinc-blende phase of MnSe [13] and MnTe [14] provides an opportunity to revisit the problem of the fcc antiferromagnet in the context of symmetry-breaking strain fields and dimensional crossover. The ideal systems for such studies are superlattices such as $\mathrm{ZnSe} / \mathrm{MnSe}$ in which thin layers of $\mathrm{MnSe}$ or MnTe may be stabilized in a variety of strain configurations. Bulk-like layers exhibit a fluctuation-induced first-order magnetic phase transition to the well-known type-III antiferromagnetic phase [4] which is characterized by a tetragonal magnetic unit cell [15]. We may then ask ourselves: how is the magnetically ordered phase affected both by strain and by a reduction in dimensionality? We find that the spin organization in such fcc antiferromagnets may be dramatically changed by simply placing them in different strain configurations, and that reduced dimensionality tends to suppress the formation of magnetic order.

We focus here on $\mathrm{ZnSe} / \mathrm{MnSe}$ and $\mathrm{ZnTe} / \mathrm{MnSe}$ superlattices in which strained zinc-blende layers of $\mathrm{MnSe}$ are sandwiched between non-magnetic layers of either $\mathrm{ZnSe}$ or $\mathrm{ZnTe}$. The non-magnetic layers are thick enough that the MnSe layers are magnetically isolated; the only reason to use superlattice structures is to provide enough magnetic material for the experimental measurements. The lattice parameter of unstrained $\mathrm{MnSe}(5.92 \AA)$ is in between that of $\mathrm{ZnSe}$ and $\mathrm{ZnTe}(5.667 \AA$ and $6.099 \AA$, respectively). Hence, the magnetic layers in $\mathrm{ZnSe} / \mathrm{MnSe}$ superlatlices are subject to an in-plane compressive strain and a perpendicular expansion, while the situation is just the opposite in $\mathrm{ZnTe} / \mathrm{MnSe}$ superlattices. The superlattices are grown by MBE on (001) GaAs wafers (typically, 2 to $3 \mathrm{~cm}^{2}$ in area), after first depositing a thick buffer layer of either ZnSe or ZnTe. The total number of superlattice periods is usually between 100 and 400 , and is adjusted so as to provide a total of about $5000 \AA$ of magnetic layer thickness. The sample surface is monitored during growth by in situ reflection high-energy electron diffraction (RIEED) at $10 \mathrm{keV}$. By monitoring oscillations in the intensity of the specular spot in the RIIEED pattern along the [011] azimuth, superlattice periods ean be precisely timed in integral numbers of monolayers.

A detailed picture of the antiferromagnetic phases in these materials is obtained from neutron diffraction experiments performed at the $20 \mathrm{MW}$ reactor at the National Institute of Standards and Technology using a triple axis spectrometer operated in the elastic diffraction mode, with an incident neutron beam of 
encrgy $14.8 \mathrm{meV}$ and angular collimation of $40^{\prime}$ throughout. Scans along the [001] direction in the vicinity of Bragg reflection positions for bulk unstrained materials produce characteristic diffraction patterns from the superlattice crystal structure, with harmonic peaks occurring at regular intervals $2 \pi / L$ in $Q$-space (where $L$ is the bilayer thickness). The period $L$ determined from these peaks is in very good agreement with that expected from RHEED oscillations. The superlattice reflections show only a slight broadening, and their deconvoluted widths indicate that the range of crystalline coherence in the samples in the growth direction as well as in the growth plane is $>500 \AA$. From a straightforward analysis of nuclear diffraction data, we are able to measure both the in-plane and out-of-plane lattice parameters ( $a$ and $c$, respectively) of the strained MnSe laycrs, and hence characterize the strain in these systems.

We first discuss the $\mathrm{ZnSe} / \mathrm{MnSe}$ system in which the MnSe layers are subject to in-plane compressive strain. At low temperatures, additional diffraction maxima are observed corresponding to type-III antiferromagnetic superstructure points (Fig. 1). The temperature behavior of these peaks clearly indicates their

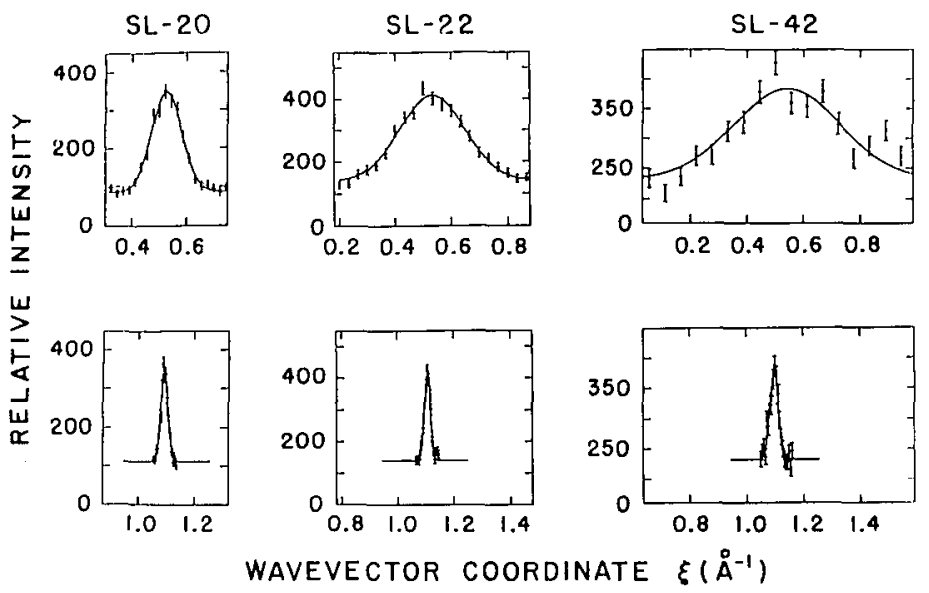

Fig. 1. Magnetic diffraction from three $\mathrm{ZnSe} / \mathrm{MnSe}$ superlattices. In the upper and lower panels, we show scans along the [001] and [010] directions, respectively, of the $(0,1,1 / 2)$ reflection. The range of antiferromagnetic order in a particular direction is inversely proportional to the width of the diffraction peak. The samples SL-20, SL-22 and SL-42 contain 15, 7 and 3 monolayers of $\mathrm{MnSe}$ in each period, respectively.

magnetic origin: namely, these peaks only appear below a characteristic Néel temperature $T_{\mathrm{N}}$ and increase in intensity as the temperature is further lowered. Unlike the case of unstrained, bulk type-III antiferromagnets where cubic symmetry allows three equivalent orientations of the type-III domain, we observe only one domain orientation, with the tetragonal axis pointing along [001] (Fig. 2). From the deconvoluted widths of scans along [010] and [100], we deduce that the antiferromagnetic order is long range within the layer plane, and is limited to the $\mathrm{MnSe}$ layer thickness along the growth direction. Further analysis of diffraction data also 


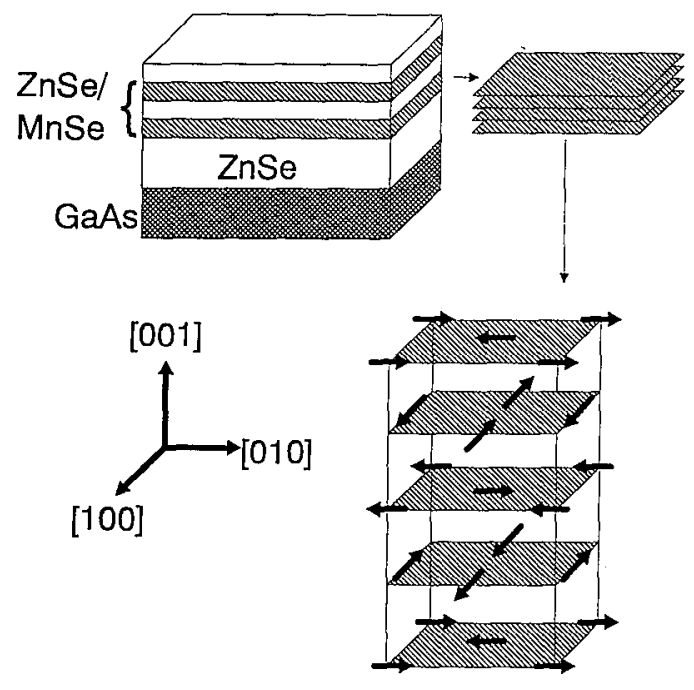

Fig. 2. Type-III magnetic unit cell in $\mathrm{ZnSe} / \mathrm{MnSe}$ superlattices, showing the unique orientation of the cell with respect to the sample geometry. The spin axis lies within the layer plane as shown, but the spins at $z=0,1 / 2$ can be rotated by an arbitrary amount relative to those at $z=1 / 4,3 / 4$. Anisotropic Dzyaloshinski-Moriya exchange [16] favors the noncollinear spin arrangement shown [17].

indicates that the spins lie within the layer plane as indicated in Fig. 2.

We now turn our attention to $\mathrm{ZnTe} / \mathrm{MnSe}$ superlattices in which the $\mathrm{MnSe}$ layers are subject to in-plane tensile strain instead of compressive strain. Once again, below a characteristic transition temperature (typically around $70 \mathrm{~K}$ ), additional diffraction peaks of magnetic character are observed. Ilowever, instead of peaks characteristic of type-III ordering, these peaks are located at $( \pm \delta, 0, l)$, $(2 \pm \delta, 0, l), \ldots$ (where $l$ is an odd integer), and at $(1 \pm \delta, l, 0),(3 \pm \delta, l, 0) \ldots$, and symmetry equivalent pairs (Fig. 3). The peaks are clearly shifted from low order commensurate lattice positions (e.g., $(2 / 3,1,0))$ and this shift is temperature-dependent (Fig. 3).

The incommensurate helimagnetic structure deduced from the diffraction data is shown in Fig. 4. There are two equivalent helical domains, directed along the [010] and [100] directions. Once again, the magnetic order is long-ranged $(>500 \AA$ ) within the layer plane, and essentially limited by the layer thickness in the growth direction. Hence, as depicted in Fig. 4, the helical domains consist of (100) (or (010)) "bands" of antiferromagnetically coupled spins, with the direction of the spins rotating by an angle $\varphi$ for successive bands. The angle $\varphi$ is related to the magnetic reflection index $\delta$ and the helix period $\Lambda$ through $\varphi / \pi=a / \Lambda=\delta$. For $\varphi=0$, which corresponds to a helix with an infinite period, this order is identical with the well-known collinear type-I structure [15], while for $\varphi=90^{\circ}$ (i.e. $\Lambda=2 a$ ) the helical structure becomes identical with the canted type-III order shown in Fig. 1. In all the samples studied so far, the angle $\varphi$ lies in the range betwcen 


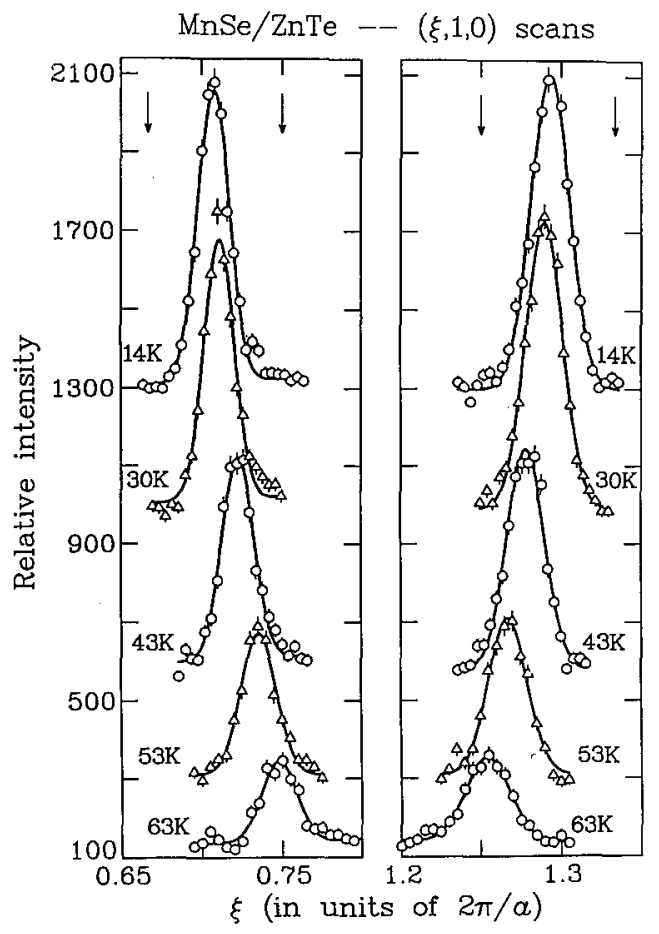

Fig. 3. Magnetic diffraction from a $\mathrm{ZnTe} / \mathrm{MnSe}$ superlattice. The scans are along the $(\xi, 1,0)$ line in reciprocal space and show pairs of magnetic reflections at $(1 \pm \delta, 1,0)$ for different temperatures below the Néel temperature.

$45^{\circ}$ and $62^{\circ}$. As the temperature is lowered from the Néel temperature, the angle increases and then saturates below $T=20 \mathrm{~K}$. We do not find any tendency for the helix period to lock-in at any low order commensurable values, as is often observed in systems which exhibit incommensurate magnetism $[18,19]$.

All the above results may be understood in a first approximation using simple mean-field arguments. The tetragonal distortion of the strained MnSe layers leads to different nearest neighbor (NN) exchange interactions parallel and perpendicular to the growth direction, denoted by $J_{1 \|}$ and $J_{1 \perp}$, respectively. When the MnSe layers are under in-plane compressive strain, the out-of-plane NN are closer than those in-plane. It is then reasonable to assume that $J_{1 \perp}>J_{1 \|}$. Ignoring next-nearest neighbor interactions, the interaction energy per spin for a type-III domain with the tetragonal axis parallel to the growth axis is then $-8 J_{1 \|}$, while that for type-III domains with the tetragonal axis within the layer plane is $-8 J_{1 \perp}$. IIence, in-plane compressive strain selects a single orientation of type-III domain, directed along the growth axis as observed.

The above analysis would predict that in-plane tensile strain should simply stabilize two equivalent type-III domains lying within the layer plane, which is not observed experimentally. A simple extension of the calculation that accounts for 


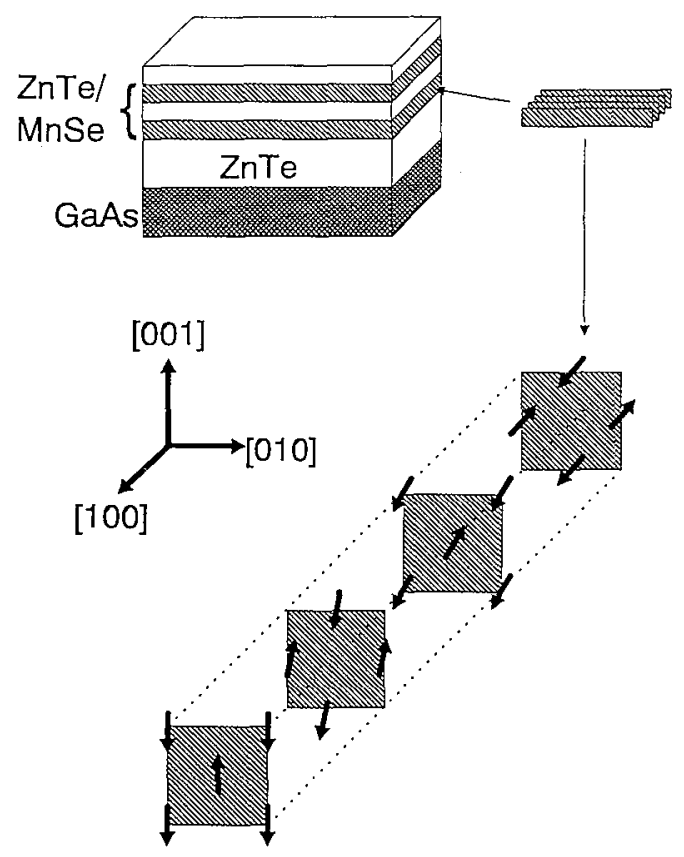

Fig. 4. Helimagnetic structure in $\mathrm{ZnTe} / \mathrm{MnSe}$ superlattices deduced from the scans in Fig. 3. The helical axis is oriented along [100] (or equivalently [010]) and hence lics within the epitaxial layer plane. Since the magnetic order is limited by the MnSe layer thickness in the growth direction but is long-ranged along [010] and [100], we obtain (100) and (010) bands of antiferromagnetically ordered spins, with the spins on adjaccnt bands rotating by an angle $\varphi$ about the helix axis. The pitch of the incommensurate helix and the angle $\varphi$ between spins in adjacent (100) planes are related to the magnetic reflection index in Fig. 3 by $\delta=a / \Lambda=\varphi / \pi$.

next-nearest-neighbor (NNN) interactions shows that in-plane tensile strain does indeed energetically favor the observed helimagnetic state over the type-III state. We find that when $0 \leq \varphi \leq 90^{\circ}$, the total magnetic energy per spin is minimized for

$$
\varphi=\arccos \left(\frac{\Delta J_{1}-\frac{1}{N} J_{1}}{2 J_{2}}\right),
$$

where $N$ is the number of magnetic monolayers constituting the MnSe layer, $J_{2}$ is the NNN exchange, and where $\Delta J_{1}=J_{1 \perp}-J_{1 \|}$. The angle $\varphi$ (and the incommensurate helical pitch) therefore depend both on the strain and on the layer thickness.

Finally, we address the role of dimensionality. Our studies of $\mathrm{ZnSe} / \mathrm{MnSe}$ superlattices indicate that while long-range in-plane order persists down to $\mathrm{MnSe}$ layer thicknesses of 3 monolayers (see Fig. 1), the Néel temperature clearly decreases with layer thickness. For example, the 3-monolayer sample shows a Néel temperature of around $75 \mathrm{~K}$ compared with $T_{\mathrm{N}}=115 \mathrm{~K}$ for a 15 -monolayer sample. 
This observation, in concert with magnetooptical evidence of paramagnetic behavior in one and two monolayers of MnSe (down to $1.5 \mathrm{~K}$ ) [13], strongly suggests that lowered dimensionality tends to suppress the formation of magnetic order. $\mathrm{A}$ heuristic analysis [6] suggests that these MnSe layers may fall into the $x y$ universality class, in which case a Kosterlitz-Thouless-like behavior may be expected on approaching $d=2$. One might then expect that the ordering temperature will be proportional to the magnetic layer thickness. Detailed measurements of exponents in the critical region are however needed to verify this conjecture. In the case of $\mathrm{ZnTe} / \mathrm{MnSe}$ superlattices, the role of finite layer thickness is already explicit in the results of the mean-field calculation (Eq. (1)). Ilowever, the situation is still unclear for $\mathrm{ZnTe} / \mathrm{MnSe}$ superlattices with very thin layers of MnSe: unlike the casc of $\mathrm{ZnSe} / \mathrm{MnSe}$ superlattices, we have not yet seen any evidence of a magnetic phase transition even samples with 5 monolayers of MnSe. Further theoretical and experimental investigations of these systems are presently underway to understand this behavior.

\section{Dynamic spin organization: magnetic polarons}

The spin organization which we discussed in the preceding section originates from $\mathrm{Mn}-\mathrm{Mn}(d-d)$ exchange interactions. Another form of co-operative spin behavior seen in diluted MS alloys is the polarization of magnetic ions by a spin-oriented population of carriers via $s p-d$ exchange. This phenomenon can be directly observed by measuring optically induced magnetization under excitation by circularly polarized light [20]. Spin-polarizcd carriers which are excited directly into impurity-bound levels maintain their spin orientation and consequently produce an exchange field that aligns magnetic ions enclosed within the Bohr orbit of the impurity-bound carrier. By combining highly sensitive SQUID techniques with femtosecond optical spectroscopy, one may monitor the dynamic behavior of these carrier-ion spin complexes (bound magnetic polarons), and hence gain direct insight into processes such as spin scattering. The availability of diluted MS quantum well structures gives us an opportunity to examine how the spin dynamics of magnetic polarons changes as the carrier wave function is "squeczed" on the length scale of the polaronic diameter and as the magnetic environment acquires a quasi-2D character.

The optically induced magnetization measurements are carried out in an integrated dc SQUID microsusceptometer [21]. The samples are placed in one pickup loop of the detector and cooled to low temperatures in a top-loading optical cryostat. When carefully shielded and immersed in superfluid ${ }^{4} \mathrm{He}$ at $T=1.5 \mathrm{~K}$, the coupled energy sensitivity of the present SQUID system corresponds to an ability to detect $10^{4}$ aligned spins. The dynamics of the spin-relaxation processes involved in optically pumped magnetization can be monitored in the time domain using a unique combination of femtosecond optical techniques and the SQUID described above. Spin-oriented carriers are first created with fiber-compressed circularly polarized optical pulses 150 fs in duration from a two-jet dye laser synchronously pumped by a frequency-doubled mode-locked Nd-doped yttrium aluminum garnet source. The time dependence of the induced magnetization can then be recorded 
by a pump-time-delayed-probe technique which takes advantage of the nonlinear magnetooptical susceptibility of the $\mathrm{Mn}^{2+}$ sublattice. Direct optical access allows true femtosecond resolution to be attained.

The above technique is employed to observe magnelic polaron dynamics in $\mathrm{ZnTe} / \mathrm{Cd}_{1-x} \mathrm{Mn}_{x}$ Se quantum wells [9]. These new MS quantum structures have two characteristics which set them apart from previously studied systems such as $\mathrm{CdTe} / \mathrm{Cd}_{1-x} \mathrm{Mn}_{x}$ Te: first, the electrons are confined to magnetic quantum wells situated between non-magnetic barriers; second, because of a deep type-II band alignment, the lowest energy hole states are confined to the $\mathrm{ZnTe}$ layers and are hence spatially separated from the magnetic layers [22]. This provides a clean experimental situation in which only spin-polarized electrons interact with the magnetic ions and electron-hole exchange effects are negligible, in contrast to the case of type-I confinement where such exchange leads to very rapid spin-flip scattering. Finally, the as-grown $\mathrm{Cd}_{1-x} \mathrm{Mn}_{x}$ Se layers have a tendency to be $n$-type, hence providing a favorable situation for the formation of electron-based magnetic polarons.

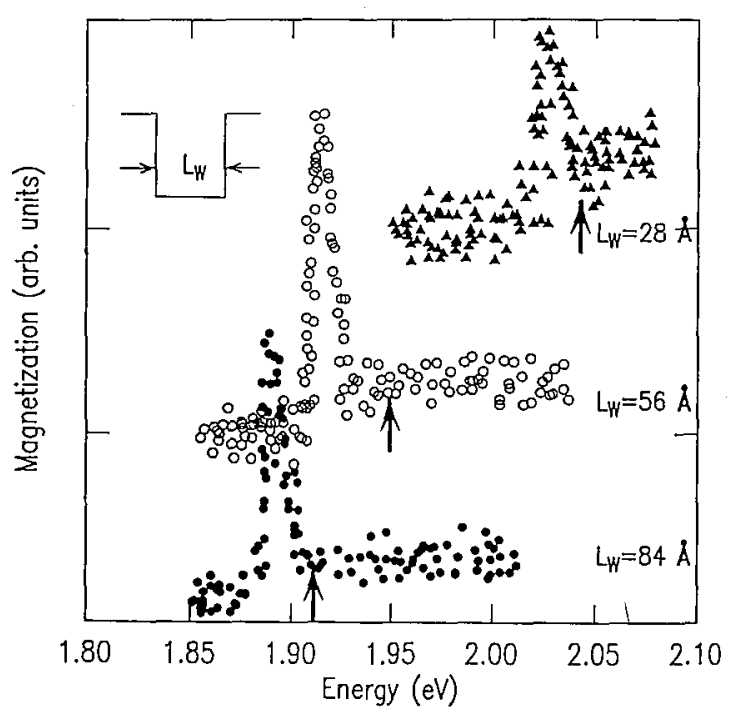

Fig. 5. Magnetic excitation spectra of three $\mathrm{ZnTe}_{\mathrm{Cd}} \mathrm{Cd}_{1-x} \mathrm{Mn}_{x} \mathrm{Se}(x=0.23)$ multiple quantum well structures having well widths of $28 \AA, 56 \AA$ and $84 \AA$, and $Z \mathrm{nTe}$ barrier thicknesses of $160 \AA$. The peaks in magnetization are the signatures of magnetic polaron formation, while the energy gaps as determined by reflectance spectroscopy are indicated by arrows.

Magnetic polaron formation in these structures is evidenced when the time-averaged magnetization induced by circularly polarized light is measured as a function of the incident photon energy, yielding a magnetic excitation spectrum analogous to more familiar photoluminescence excitation or excitonic absorption spectra (Fig. 5). A well-defined peak due to magnetic polaron formation is observed 
at an energy which is $10-25 \mathrm{meV}$ below the lowest excitonic transition seen in photoreflectance and absorption. Both the magnitude of the energy shift of the polaronic peak with respect to the $n=1$ level and its variation with quantum well width are consistent with the trend found for quantum-confined hydrogenic donors. We hence attribute the peak in the magnetic excitation spectra to the formation of electron-based polarons when carriers are directly promoted into the lowest confined donor level. For higher incident photon energies, memory of the initial spin orientation is lost through spin-flip scattering during energy relaxation. When these carriers are eventually trapped into donor-bound orbits, they form randomly oriented polarons which do not contribute significantly to the magnetic signal. This results in a decrease in the optically induced magnetization, and leaves the resonance-like peak in the dc magnetic excitation spectrum. The spectra shown in Fig. 5 are the first direct evidence for the formation of magnetic polarons in a quantum well geometry and are an elegant indication of how electronic confinement can be mapped using a magnetic probe.

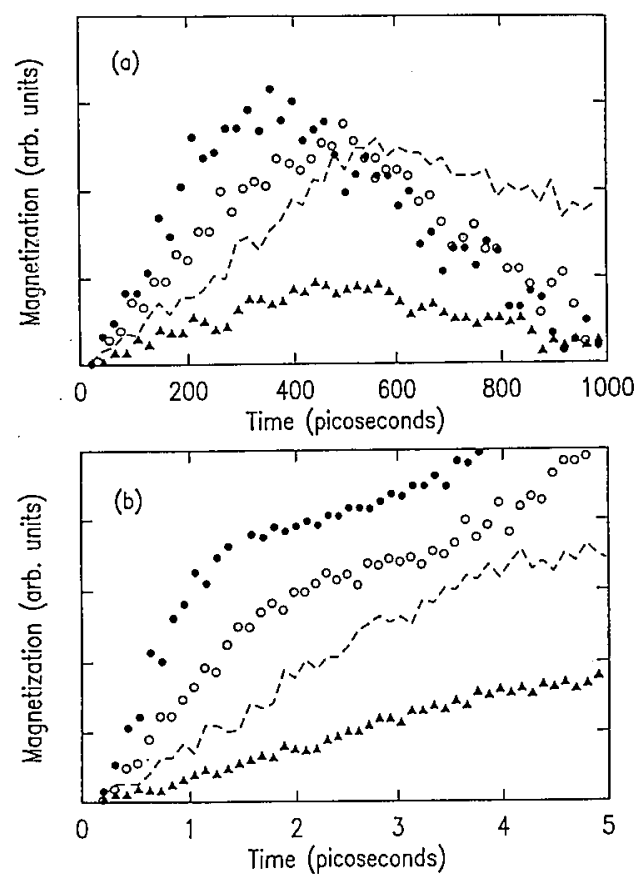

Fig. 6. Time-dependence of the optically-induced magnetization with pulsed excitation at the peak polaron energies determined from the static spectra in Fig. 5 . In (a), we observe the polaronic life-cycle. In (b), we show the initial magnetic response to the 150 fs excitation pulse. Solid circles, open circles and solid triangles represent the 84 , 56 and $28 \AA$ well samples of Fig. 1, while the dashed line is a measurement of an $84 \AA$, sample with $x=0.13$. 
Time-resolved measurements at femtosecond resolution provide a real-time picture of the spin-relaxation path that eventually produces the dc spectra. When the excitation energy coincides with the peak in the dc spectra, the time evolution of the magnetization signal directly images the polaronic life cycle, showing the growth of the magnetization over a time scale of hundreds of picoseconds as $\mathrm{Mn}^{2+}$ ions relax to produce spin-aligned clusters around donor sites (Fig. 6). The polaronic magnetization eventually decays according to the dynamics of this localized spin complex. If we monitor the time evolution of the magnetization for excitation in the high-energy plateau region of the dc spectra, we obtain dramatically different behavior (Fig. 7). In Fig. 7b, which depicts the first few picoseconds following the excitation, there is a rapid rise in the magnetization due to the transfer of angular momentum from spin-polarized carriers to the magnetic ions through spin-flip scattering. The picosecond-scale times are consistent with theoretical expectations for spin-flip scattering in II-VI MS systems. Long after the electronic spins have depolarized (Fig. 7a), we observe the spin-lattice relaxation of the magnetization.
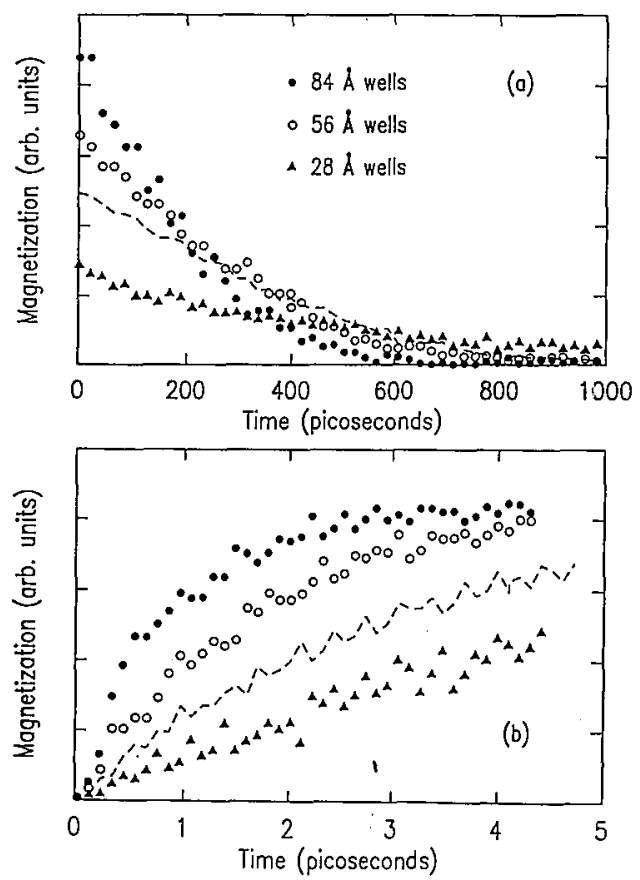

Fig. 7. Time-dependent magnetization in the samples described in Fig. 6 for above-gap excitation energies. In (a), we observe the spin-lattice relaxation of the magnetization. In (b), we see the initial transfer of angular momentum from spin-polarized carriers to magnetic ions through spin-flip scattering.

The effect of dimensionality on spin dynamics is evident in Figs. 6 and 7 in the variation of both the spin-lattice relaxation times and spin-flip scattering 
times with the magnetic layer thickness. This can be qualitatively described by a decrease in the effective magnetic concentration with decreasing layer thickness, as the magnetic ions near the interfaces see fewer neighbors than those in the center. A comparison between samples with identical layer thicknesses but with different magnetic ion concentration ( $x=0.23$ versus $x=0.13$ in Figs. 6 and 7 ) bears out this qualitative argument. Finally, detailed analysis of the time-resolved spectra also indicates how the shape of the polaron changes with quantum confinement and reduced dimensionality. Since the growth and decay of the polaron magnetization is controlled by the spin-lattice relaxation time, we can estimate the peak magnetization that would occur in Fig. 6 if the polarons were allowed to evolve to completion (rather than be limited by the carrier lifetime). In doing so, we find that the in-plane diameter remains constant at the bulk value (about $90 \AA$ ) independent of the well width, while the out-of-plane diameter clearly must shrink with decreasing well thickness. The polaron shape hence changes from a spherical structure to an ellipsoid as the wave function is compressed along the normal to the layer planes.

\section{Summary}

We have shown how the static and dynamic organization of localized magnetic spins may be experimentally observed in both diluted and undiluted MS nanostructures. These structures provide new ways in which to manipulate co-operative spin phenomena in magnetic systems through control of strain, dimensionality and quantum confinement. Commensurate as well as incommensurate antiferromagnetic states may be strain-engineered in a single magnetic system such as MnSe. Finally, the direct observation of magnetic polarons in MS quantum wells provides a powerful localized magnetic probe of dynamic spin processes in quantum geometries.

We would like to acknowledge the contributions of the following to this work: M. Freeman, P. Kłosowski, B.E. Larson and J.J. Rhyne. This work was supported by National Science Foundation Grants DMR-8821635 and DMR-8520866, and by ONR Grant N-00014-90-J-1782.

\section{References}

[1] C. Weisbuch, B. Vinter, Quantum Semiconductor Structures, Academic Press, San Diego 1991.

[2] Magnetic Surfaces, Thin Films, Multilayers, Eds. S.S.P. Parkin, H. Hopster, J.P. Renard, T. Shinjo, W. Zinn, Materials Research Sociely Symposia Proceeding, Vol. 231, MRS, Pittsburgh 1992.

[3] N. Samarth, J.K. Furdyna, Proc. of the IEEE 78, 990 (1990).

[4] P. Klosowski, T.M. Giebultowicz, J.J. Rhyne, N. Samarth, H. Luo, J.K. Furdyna, J. Appl. Phys. 70, 6221 (1991).

[5] D.D. Awschalom, J.M. Hong, L.L. Chang, G. Grinstein, Phys. Rev. Lett. 59, 1733 (1987).

[6] N. Samarth, P. Klosowski, H. Luo, T. Giebultowicz, J.K. Furdyna, J.J. Rhyne, B.E. Larson, N. Otsuka, Phys. Rev. B 44, 4701 (1991). 
[7] T. Giebułtowicz, P. Kłosowski, J.J. Rhyne, N. Samarth, II. Luo, J.K. Furdyna, in: Proc. Int. Conf. Neutron Scattering, Oxford (UK) 1991.

[8] P. Kłosowski, T. Giebułtowicz, N. Samarth, H. Luo, J.K. Furdyna, J.J. Rhyne, J. Magn. Magn. Mater. 104-107, 1795 (1992).

[9] D.D. Awschalom, M.R. Freeman, N. Samarth, H. Luo, J.K. Furdyna, Phys. Rev. Lett. 66, 1212 (1991).

[10] N. Dai, H. Luo, F.C. Zhang, N. Samarth, M. Dobrowolska, J.K. Furdyna, Phys. Rev. Lett. 67, 3824 (1991); W.C. Chou, A. Petrou, J. Warnock, B.T. Jonker, ibid. 67, 3820 (1991).

[11] D. ter Haar, M. Lines, Philos. Trans. R. Soc. Lond. A 254, 521 (1962).

[12] A.J. Annila, K.N. Clausen, A.S. Oja, J.T. Tuoriniemi, H. Weinfurter, Phys. Rev. B 45, 7772 (1992).

[13] L.A. Kolodziejski, R.L. Gunshor, N. Otsuka, B.P. Gu, Y. Hefetz, A.V. Nurmikko, Appl. Phys. Lett. 48, 1482 (1986).

[14] S.M. Durbin, J. Han, Sungki O, M. Kobayashi, D.R. Menke, R.L. Gunshor, Q. Fu, N. Pelekanos, A.V. Nurmikko, D. Li, J. Gonsalves, N. Otsuka, A ppl. Phys. Lett. 55, 2087 (1989).

[15] J. Smart, Effective Field Theories of Magnetism, W.D. Saunders Comp., Philadelphia 1966.

[16] B.E. Larson, K.C. Hass, H. Ehrenreich, A.E. Carlsson, Phys. Rev. B 37, 4137 (1988).

[17] F. Keffer, Phys. Rev. 126, 896 (1962).

[18] P. Bak, Rep. Prog. Phys. 45, 587 (1982).

[19] Yu.A. Izyumov, Physica B 174, 9 (1991).

[20] D.D. Awschalom, J. Warnock, S. von Molnar, Phys. Rev. Lell. 58, 812 (1987).

[21] D.D. Awschalom, J.R. Rozen, M.B. Ketchen, W.J. Gallagher, A.W. Kleinsasser, R.L. Sandstrom, B. Bumble, Appl. Phys. Lett. 53, 2108 (1988).

[22] N. Samarth et al., J. Vac. Sci. Technol. B 10, 915 (1992). 